\title{
Nonnegative solutions of some quasilinear elliptic inequalities and applications
}

\author{
Lorenzo D’Ambrosio* and Enzo Mitidieri ${ }^{\dagger}$ \\ May 72009 \\ Accepted for publication on \\ Mat. Sb., 2010, Vol. 201
}

\begin{abstract}
Let $f: \mathbb{R} \rightarrow \mathbb{R}$ be a continuous function. We prove that under some additional assumptions on $f$ and $A: \mathbb{R} \rightarrow \mathbb{R}_{+}$, weak $\mathscr{C}^{1}$ solutions of the differential inequality $-\operatorname{div}(A(|\nabla u|) \nabla u) \geq f(u)$ on $\mathbb{R}^{N}$ are nonnegative. Some extensions of the result in the framework of subelliptic operators on Carnot Groups are considered.
\end{abstract}

\section{Introduction}

In this paper we shall study the following problem.

Let $L$ be a second order differential operator and let $f: \mathbb{R} \rightarrow \mathbb{R}$ be a continuous function. Find additional assumptions on $(L, f)$ that imply the positivity of the possible solutions of the differential inequality

$$
L(u) \geq f(u) \quad \text { on } \quad \mathbb{R}^{N} .
$$

Some partial answers to this problem have been obtained in [10, 16]. In those papers, the authors deal with elliptic inequalities of the form (1.1) in the case when $L$ is the Laplacian operator or the polyharmonic operator $(-\Delta)^{k}$ in the Euclidean setting or, more generally $L$ is a sub elliptic Laplacian on a Carnot group and $f$ is nonnegative. The main strategy used in [10, 16] for proving positivity results was via integral representation formulae.

\footnotetext{
${ }^{*}$ Dipartimento di Matematica, via E. Orabona, 4 - Università degli Studi di Bari, I-70125 Bari, Italy dambros@dm.uniba.it

${ }^{\dagger}$ Dipartimento di Matematica e Informatica, via A. Valerio, 12/1 - Università degli Studi di Trieste, I-34127 Trieste, Italy mitidier@units .it
} 
One essential difficulty using this approach is that no assumptions on the behavior of the solutions at infinity are known. A typical example in this direction is given by,

$$
-\Delta u \geq|u|^{q} \quad \text { on } \quad \mathbb{R}^{N}
$$

where $N \geq 3$ and $q>1$.

The following result holds (see [10]).

Theorem 1.1 Let $N \geq 3$ and $q>1$. Let $u \in L_{l o c}^{q}\left(\mathbb{R}^{N}\right)$ be a distributional solution of (1.2) and let Leb(u) be the set of its Lebesgue points. If $x \in \operatorname{Leb}(u)$, then

$$
u(x) \geq C_{N} \int_{\mathbb{R}^{N}} \frac{|u(y)|^{q}}{|x-y|^{N-2}} d y
$$

where $C_{N}$ is an explicit positive constant.

From this result it follows that, if $u$ is a solution of (1.2) then, either $u(x)=0$ or $u(x)>0$ a.e. on $\mathbb{R}^{N}$.

Obviously, the approach via representation formulae cannot be applied to quasilinear problems. In this paper we shall consider a class of quasilinear model problems for which the positivity property mentioned at the beginning of this introduction holds.

More precisely, we shall deal with the case when $L$ is the $p$-Laplacian operator, namely $\Delta_{p} \cdot=\operatorname{div}\left(|\nabla \cdot|^{p-2} \nabla \cdot\right)$, or the mean curvature operator $-\operatorname{div}\left(\frac{\nabla \cdot}{\sqrt{1+|\nabla \cdot|^{2}}}\right)$. In this cases some results on nonnegativity of solutions of (1.1) are proved by using a suitable comparison Lemma (see Lemma 2.15 below). We will apply this results to the problem of a priori bounds of solutions and nonexistence theorems. For interesting results on related coercive equations see the very recent paper [14].

This paper is organized as follows. In section 2 we state and prove our main results in the Euclidean setting for the $p$-Laplacian and the mean curvature operator. In addition we point out some consequences and briefly discuss the sharpness of some of the results.

In section 3 we briefly indicate some generalizations to other quasilinear operators and to differential inequalities on Carnot groups. We end the paper with an appendix which contains some well known facts on Carnot groups.

A shorter version of this paper appeared in [9]. After this paper was submitted for publication (May 2009), we learned form Professor James Serrin that in the Euclidean setting, related results similar to Lemma 2.15] are contained in [21]. See in particular Sections 2.2 and 2.4 and also Chapter 3. It seems likely that suitable versions of the comparison principles contained in [21] still hold in the framework of Carnot groups. This will be investigated in a forthcoming paper. 


\section{Main results}

Throughout this paper we shall assume that $N \geq 2$ and we deal with weak $\mathscr{C}^{1}$ solutions of the problems under consideration. See Definition 2.14 below for further details. Our main results are the following.

Theorem 2.1 Let $p>1$ and $N>1$. Let $f: \mathbb{R} \rightarrow \mathbb{R}$ be a continuous function such that

$$
f(t)>0 \quad \text { if } t<0, \quad f \quad \text { is non increasing on }]-\infty, 0[
$$

and

$$
\int_{-\infty}^{-1}\left(\int_{t}^{-1} f(s) d s\right)^{-\frac{1}{p}} d t<+\infty
$$

If $u$ is a solution of

$$
-\operatorname{div}\left(|\nabla u|^{p-2} \nabla u\right) \geq f(u) \quad \text { on } \quad \mathbb{R}^{N},
$$

then $u \geq 0$ on $\mathbb{R}^{N}$. Moreover if $f(t) \geq 0$ for $t \geq 0$ then, either $u \equiv 0$ or $u>0$ on $\mathbb{R}^{N}$.

Corollary 2.2 Let $p>1$. Let $f: \mathbb{R} \rightarrow \mathbb{R}$ be a continuous function such that $f(t) \geq C|t|^{q}$ for $t<0$. Let $u$ be a solution of

$$
-\operatorname{div}\left(|\nabla u|^{p-2} \nabla u\right) \geq f(u) \quad \text { on } \quad \mathbb{R}^{N} .
$$

If $q>p-1$ then $u \geq 0$ on $\mathbb{R}^{N}$. Moreover if $f(t) \geq 0$ for $t \geq 0$ then, either $u \equiv 0$ or $u>0$ on $\mathbb{R}^{N}$.

In the case of the mean curvature operator the above results can be improved. Indeed, the claim follows without the assumption (2.4) on $f$.

Theorem 2.3 Let $f: \mathbb{R} \rightarrow \mathbb{R}$ be a continuous function satisfying (2.3). Let u be a solution of

$$
-\operatorname{div}\left(\frac{\nabla u}{\sqrt{1+|\nabla u|^{2}}}\right) \geq f(u) \quad \text { on } \quad \mathbb{R}^{N} .
$$

Then $u \geq 0$ on $\mathbb{R}^{N}$.

A first consequence of the above results is the following a priori estimate.

Theorem 2.4 Let $p>1$ and $N>1$. Let $f: \mathbb{R} \rightarrow \mathbb{R}$ be a continuous function such that there exists $\alpha, \beta \in \mathbb{R}, \alpha \leq \beta$ such that

$f_{]-\infty, \alpha[}$ is positive and non increasing, $f_{] \beta,+\infty[}$ is negative and non increasing, (2.8) 
and

$$
\int_{-\infty}^{\alpha}\left(\int_{t}^{\alpha} f(s) d s\right)^{-\frac{1}{p}} d t<+\infty, \quad \int_{\beta}^{\infty}\left(\int_{\beta}^{t}-f(s) d s\right)^{-\frac{1}{p}} d t<+\infty .
$$

If $u$ is a solution of

$$
-\operatorname{div}\left(|\nabla u|^{p-2} \nabla u\right)=f(u) \quad \text { on } \quad \mathbb{R}^{N},
$$

then $u$ is bounded and $\alpha \leq u(x) \leq \beta$ for any $x \in \mathbb{R}^{N}$.

Again, for the mean curvature operator we can require more general assumption on $f$.

Theorem 2.5 Let $f: \mathbb{R} \rightarrow \mathbb{R}$ be a continuous function such that

$$
\liminf _{t \rightarrow-\infty} f(t)>0 .
$$

If $u$ is a solution of (2.7), then $f$ has at least a zero, and set $\alpha:=$ the first zero of $f$ (that is $\alpha:=\min S$ where $S:=f^{-1}(0)$ ) we have $u \geq \alpha$. In particular if $f>0$ the (2.7) has no solution.

Moreover if

$$
\limsup _{t \rightarrow+\infty} f(t)<0
$$

and $u$ solves

$$
-\operatorname{div}\left(\frac{\nabla u}{\sqrt{1+|\nabla u|^{2}}}\right)=f(u) \text { on } \mathbb{R}^{N} .
$$

then $u$ is bounded and $\alpha \leq u(x) \leq \beta$ for any $x \in \mathbb{R}^{N}$, where $\beta:=$ last zero of $f$ (that is $\beta:=\max S$ ).

A direct consequence of Theorems 2.4 and 2.3, 2.5 are the following Liouville theorems.

Corollary 2.6 Let $p>1$ and $N>1$. Let $f: \mathbb{R} \rightarrow \mathbb{R}$ be a non increasing continuous function such that

$$
f(t)>0 \text { if } t<0 \text {, and } f(t)<0 \text { if } t>0 \text {, }
$$

and

$$
\int_{-\infty}^{-1}\left(\int_{t}^{-1} f(s) d s\right)^{-\frac{1}{p}} d t<+\infty, \quad \int_{1}^{\infty}\left(\int_{1}^{t}-f(s) d s\right)^{-\frac{1}{p}} d t<+\infty .
$$

If $u$ is a solution of

$$
-\operatorname{div}\left(|\nabla u|^{p-2} \nabla u\right)=f(u) \quad \text { on } \quad \mathbb{R}^{N},
$$


then $u \equiv 0$ on $\mathbb{R}^{N}$.

In particular, if $q>p-1$ and $u$ is a solution of

$$
\Delta_{p} u=|u|^{q-1} u \quad \text { on } \quad \mathbb{R}^{N},
$$

then $u \equiv 0$ on $\mathbb{R}^{N}$.

Remark 2.7 The conclusion for equation (2.15) in the case $p=2$ has been proved by Brezis [3]. An important generalization, by using a different technique, in the Euclidean case, has been obtained by Serrin [23, Theorem 2]. For a version of Corollary 2.6] in the framework of Carnot groups see Theorem 3.12 below.

Theorem 2.8 Let $p>1$ and $N>1$. Let $f: \mathbb{R} \rightarrow \mathbb{R}$ be a positive, non increasing, continuous function satisfying (2.4). Then the inequality (2.5) has no solutions.

Theorem 2.9 Let $N>1$. Let $f: \mathbb{R} \rightarrow \mathbb{R}$ be a non increasing, continuous function and $f \not \equiv 0$. If $u \in C^{2}\left(\mathbb{R}^{N}\right)$ is a solution of

$$
-\operatorname{div}\left(\frac{\nabla u}{\sqrt{1+|\nabla u|^{2}}}\right)=f(u) \quad \text { on } \quad \mathbb{R}^{N}
$$

then $u$ is constant, that is $u \equiv \alpha$ and $f(\alpha)=0$. In particular if $f(t) \neq 0$ for any $t$, then (2.16) has no solutions.

In addition, if $f$ is supposed to be positive then the inequality

$$
-\operatorname{div}\left(\frac{\nabla u}{\sqrt{1+|\nabla u|^{2}}}\right) \geq f(u) \text { on } \mathbb{R}^{N}
$$

has no solutions.

Remark 2.10 For a different proof of the first part of Theorem 2.9 see [13]. The claim concerning the inequality (2.17) is new and of independent interest.

The following results are an easy consequence of the fact that the only nonnegative functions $u$ such that $-\Delta_{p} u \geq 0$ on $\mathbb{R}^{N}$ with $N \leq p$ or $-\operatorname{div}\left(\frac{\nabla u}{\sqrt{1+|\nabla u|^{2}}}\right) \geq 0$ on $\mathbb{R}^{2}$ are the constants, see [11].

Corollary 2.11 Let $p \geq N>1$ and $f: \mathbb{R} \rightarrow[0,+\infty[$ be a continuous function satisfying (2.3) and (2.4). If $u$ is a solution of (2.5) then $u$ is constant on $\mathbb{R}^{N}$. More precisely $u \equiv \alpha \geq 0$ and $f(\alpha)=0$.

Moreover if $f(t)>0$ for $t \geq 0$, then (2.5) has no solutions. 
Corollary 2.12 Let $f: \mathbb{R} \rightarrow[0,+\infty$ [ be a continuous function satisfying (2.3). Let $u$ be a solution of

$$
-\operatorname{div}\left(\frac{\nabla u}{\sqrt{1+|\nabla u|^{2}}}\right) \geq f(u) \quad \text { on } \quad \mathbb{R}^{2} .
$$

Then $u$ is constant on $\mathbb{R}^{2}$. More precisely $u \equiv \alpha \geq 0$ and $f(\alpha)=0$.

Moreover if $f(t)>0$ for $t \geq 0$, then (2.18) has no solutions.

Remark 2.13 The above assumptions on $f$ are sharp in the following sense. If $p=2$ and $q=1=p-1$ the result is false. Indeed the equation

$$
-\Delta u=|u| \quad \text { on } \quad \mathbb{R}^{N}
$$

admits the explicit negative solution

$$
u(x):=-\operatorname{Exp}\left(x_{1}\right), \quad x \in \mathbb{R}^{N},
$$

or solutions that changes sign (see [10]).

In the general case $q=p-1$ the equation

$$
\Delta_{p} u=u^{p-1} \quad \text { on } \quad \mathbb{R}^{N}
$$

admits a positive solution (see for instance [17]). Therefore, the equation

$$
-\Delta_{p} u=|u|^{p-1} \quad \text { on } \quad \mathbb{R}^{N}
$$

has a negative solution.

Let us briefly describe the idea of the proof of our main result. Let $u$ be a solution of (2.5). Without loss of generality we will show that $u(0) \geq 0$. The function $U:=-u$ satisfies the inequality

$$
\operatorname{div}\left(|\nabla U|^{p-2} \nabla U\right) \geq f(-U) \quad \text { on } \quad \mathbb{R}^{N}
$$

Let $v$ be a positive solution of

$$
\operatorname{div}\left(|\nabla v|^{p-2} \nabla v\right)=f(-v) \quad \text { on } \quad B_{R}
$$

such that $v(0)=a>0$ and $v(x) \rightarrow+\infty$ as $|x| \rightarrow R$. The assumptions on $f$ imply the existence of $v$. Since $U(x) \leq v(x)$ for $|x|$ close to $R$, by a comparison Lemma (see Lemma below) it follows that $U(x) \leq v(x)$ for any $|x|<R$. In particular $U(0) \leq v(0)=a$. Letting $a \rightarrow 0$ we have $U(0) \leq 0$. Hence $u(0) \geq 0$. Finally, if $f(t) \geq 0$ for $t \geq 0$, by the weak Harnack inequality we get that, either $u \equiv 0$ or $u>0$ on $\mathbb{R}^{N}$. 


\subsection{A comparison Lemma}

In this section, we shall prove a comparison Lemma. This Lemma will be useful when dealing with more general operators then the $p$-Laplacian and the mean curvature operator, thus we shall present it in a general form. To this end let us introduce some notations. Let $\mu=\left(\mu(x)_{i, j}\right)$ be a matrix with $N$ columns and $l(\leq N)$ rows with entries belonging, for simplicity, to $\mathscr{C}^{1}\left(\mathbb{R}^{N}\right)$. We denote by $\nabla_{L}:=\mu \nabla$ and by $\operatorname{div}_{L}=-\nabla_{L}^{*}=\operatorname{div}\left(\mu^{T} \cdot\right)$. The isotropic Euclidean case corresponds to the choice $\mu=I_{N}$ the unitary matrix of dimension $N$.

We shall assume that if $\nabla_{L} u=0$ on a connected region $\Omega$ then $u \equiv$ const in such a region.

Definition 2.14 Let $\Omega \subset \mathbb{R}^{N}$ be an open set, let $A: \mathbb{R} \rightarrow \mathbb{R}$ and $h: \Omega \rightarrow \mathbb{R}$ be a continuous functions. We say that $u$ is a solution of

$$
\operatorname{div}_{L}\left(A\left(\left|\nabla_{L} u\right|\right) \nabla_{L} u\right) \geq h \quad \text { on } \quad \Omega
$$

if $u \in \mathscr{C}^{1}(\Omega)$ and for any nonnegative $\phi \in \mathscr{C}_{0}^{1}(\Omega)$, we have

$$
-\int_{\Omega} A\left(\left|\nabla_{L} u\right|\right) \nabla_{L} u \cdot \nabla_{L} \phi \geq \int_{\Omega} h \phi .
$$

In a similar manner we can define solutions of the inequalities

$$
-\operatorname{div}_{L}\left(A\left(\left|\nabla_{L} u\right|\right) \nabla u\right) \geq h \text { and } \operatorname{div}_{L}\left(A\left(\left|\nabla_{L} u\right|\right) \nabla u\right) \leq h .
$$

The following Lemma is useful when considering solutions of inequalities of the form,

$$
\operatorname{div}_{L}\left(A\left(\left|\nabla_{L} u\right|\right) \nabla_{L} u\right) \geq g_{1}(x, u) \quad \text { on } \quad \Omega
$$

and

$$
\operatorname{div}_{L}\left(A\left(\left|\nabla_{L} v\right|\right) \nabla_{L} v\right) \leq g_{2}(x, v) \quad \text { on } \quad \Omega
$$

Here, $A$ is a continuous function such that $A(t)>0$ for $t>0$ and for $i=1,2, g_{i}: \Omega \times \mathbb{R} \rightarrow \mathbb{R}$ is continuous.

Lemma 2.15 Let $\Omega$ be a bounded open set and let $u$ and $v$ be solutions of (2.19) and (2.20) respectively. Assume that

1. (a) For any $x \in \Omega, t \geq s \geq 0$ there holds $g_{1}(x, t) \geq g_{2}(x, s), g_{1}(x, \cdot)$ is not decreasing on $] 0,+\infty[$ and $v \geq 0$;

or 
(b) For any $x \in \Omega, t \geq s$ there holds $g_{1}(x, t) \geq g_{2}(x, s)$ and $g_{1}(x, \cdot)$ is not decreasing;

2. The function $t A(t)$ is increasing and positive for $t>0$;

3. $u \leq v$ on $\partial \Omega$.

Then $u \leq v$ on $\Omega$.

Proof . Let $u$ and $v$ be solutions of (2.19) and (2.20) respectively. Let $\epsilon>0$ be fixed and set $v_{\epsilon}:=v+\epsilon$. It is a simple matter to check that the function $v_{\epsilon}$ satisfies the inequality

$$
\operatorname{div}_{L}\left(A\left(\left|\nabla_{L} w\right|\right) \nabla_{L} w\right) \leq g_{1}(x, w) \quad \text { on } \quad \Omega
$$

Therefore, for any nonnegative $\phi \in \mathscr{C}_{0}^{1}(\Omega)$ we have

$$
-\int_{\Omega}\left(A\left(\left|\nabla_{L} u\right|\right) \nabla_{L} u-A\left(\left|\nabla_{L} v_{\epsilon}\right|\right) \nabla_{L} v_{\epsilon}\right) \cdot \nabla_{L} \phi \geq \int_{\Omega}\left(g_{1}(x, u)-g_{1}\left(x, v_{\epsilon}\right)\right) \phi .
$$

Next we choose $\phi$ as follows: $\phi:=\left(\left(u-v_{\epsilon}\right)_{+}\right)^{2}$. It is clear that $\phi$ is nonnegative and $\phi \in \mathscr{C}^{1}(\Omega)$. Moreover, since $v_{\epsilon}-u \geq \epsilon>0$ on $\partial \Omega$, it follows that $\phi$ has compact support. Substituting $\phi$ in (2.21), we obtain

$$
-\int_{\Omega}\left(A\left(\left|\nabla_{L} u\right|\right) \nabla_{L} u-A\left(\left|\nabla_{L} v\right|\right) \nabla_{L} v\right) \cdot\left(\nabla_{L} u-\nabla_{L} v\right) 2\left(u-v_{\epsilon}\right)_{+} \geq \int_{\Omega}\left(g_{1}(x, u)-g_{1}\left(x, v_{\epsilon}\right)\right) \phi .
$$

We claim that

$$
\left(A\left(\left|\nabla_{L} u\right|\right) \nabla_{L} u-A\left(\left|\nabla_{L} v\right|\right) \nabla_{L} v\right) \cdot\left(\nabla_{L} u-\nabla_{L} v\right) \geq 0 .
$$

Indeed,

$$
\begin{aligned}
& \left(A\left(\left|\nabla_{L} u\right|\right) \nabla_{L} u-A\left(\left|\nabla_{L} v\right|\right) \nabla_{L} v\right) \cdot\left(\nabla_{L} u-\nabla_{L} v\right) \\
& =A\left(\left|\nabla_{L} u\right|\right)\left|\nabla_{L} u\right|^{2}+A\left(\left|\nabla_{L} v\right|\right)\left|\nabla_{L} v\right|^{2}-\left(A\left(\left|\nabla_{L} u\right|\right)+A\left(\left|\nabla_{L} v\right|\right)\right)\left(\nabla_{L} u \cdot \nabla_{L} v\right) \\
& =\left(A\left(\left|\nabla_{L} u\right|\right)\left|\nabla_{L} u\right|-A\left(\left|\nabla_{L} v\right|\right)\left|\nabla_{L} v\right|\right)\left(\left|\nabla_{L} u\right|-\left|\nabla_{L} v\right|\right)+ \\
& \quad+\left(A\left(\left|\nabla_{L} u\right|\right)+A\left(\left|\nabla_{L} v\right|\right)\right)\left(\left|\nabla_{L} u\right|\left|\nabla_{L} v\right|-\nabla_{L} u \cdot \nabla_{L} v\right)=: I_{1}+I_{2} .
\end{aligned}
$$

Since $A \geq 0$, we have $I_{2} \geq 0$. From the monotonicity of $t A(t)$, it follows that

$$
I_{1}=\left(A\left(\left|\nabla_{L} u\right|\right)\left|\nabla_{L} u\right|-A\left(\left|\nabla_{L} v\right|\right)\left|\nabla_{L} v\right|\right)\left(\left|\nabla_{L} u\right|-\left|\nabla_{L} v\right|\right) \geq 0 .
$$


Assume first that $g_{1}(x,$.$) is strictly increasing. Therefore, the inequality \left(g_{1}(x, u)-\right.$ $g_{1}\left(x, v_{\epsilon}\right)\left(\left(u-v_{\epsilon}\right)_{+}\right)^{2} \geq 0$ holds for every $x \in \Omega$. As a consequence, $\left(g_{1}(x, u)-g_{1}\left(x, v_{\epsilon}\right)((u-\right.$ $\left.\left.v_{\epsilon}\right)_{+}\right)^{2}=0$ on $\Omega$ and hence $u \leq v_{\epsilon}$.

This completes the proof in case $g_{1}(x,$.$) is strictly increasing. For the general case we$ need of an extra argument. Indeed, from (2.22) and (2.23) we have that $\int_{\Omega}\left(I_{1}+I_{2}\right)(u-$ $\left.v_{\epsilon}\right)_{+}=0$. Let $x \in \Omega$ be such that $u(x) \geq v_{\epsilon}(x)$. Since $I_{1} \geq 0$ and $I_{2} \geq 0$ we have $I_{1}(x)=0=I_{2}(x)$.

We claim that $\nabla_{L} u(x)=\nabla_{L} v(x)$. Indeed, if $\nabla_{L} u(x) \neq \nabla_{L} v(x)$, from $I_{2}(x)=0$, we deduce that $\left|\nabla_{L} u(x)\right| \neq\left|\nabla_{L} v(x)\right| 1$. Thus from $I_{1}(x)=0$, the fact that $t A(t)$ is injective, it follows that

$$
0=A\left(\left|\nabla_{L} u\right|\right)\left|\nabla_{L} u\right|-A\left(\left|\nabla_{L} v\right|\right)\left|\nabla_{L} v\right| \neq 0 .
$$

This implies $\nabla_{L}\left(\left(u-v_{\epsilon}\right)_{+}\right)^{2}=0$ on $\Omega$, that is $\left(\left(u-v_{\epsilon}\right)_{+}\right)^{2}=\phi=0$.

Therefore, letting $\epsilon \rightarrow 0$ in $u \leq v+\epsilon$ the claim follows.

Remark 2.16 It is possible to extend the above result to situations where the function $A$ depends on $x$. Namely, when $A: \Omega \times \mathbb{R} \rightarrow \mathbb{R}$ is continuous and for any $x \in \Omega$ the function $t \in] 0,+\infty[\rightarrow t A(x, t)$ is increasing and strictly positive.

\subsection{Proofs of the Main Results}

The proof of Theorem 2.1 relies on the following.

Theorem 2.17 Let $g: \mathbb{R} \rightarrow \mathbb{R}$ be a continuous function such that, $g(t)>0$ if $t<0, g$ is non decreasing on $] 0,+\infty[$, and

$$
\int_{1}^{+\infty}\left(\int_{1}^{t} g(s) d s\right)^{-\frac{1}{p}} d t<+\infty .
$$

For any $p>1, a>0, D>1$, there exists a function $\varphi$ and $R>0$ such that, $\varphi$ is a solution of

$$
\left(r^{D-1}\left|\varphi^{\prime}(r)\right|^{p-2} \varphi^{\prime}(r)\right)^{\prime}=r^{D-1} g(\varphi(r)), \quad \varphi(0)=a, \quad \varphi^{\prime}(0)=0,
$$

$\phi$ is increasing on $] 0, R[$ and $\varphi(r) \rightarrow+\infty$ as $r \rightarrow R$.

See [20] for a proof in the case $p=2$ and [17] for the quasilinear case $p \neq 2$.

Proof of Theorem 2.1. Let $u$ be a solution of (2.5). Since the inequality is invariant under translations, it is sufficient to prove that $u(0) \geq 0$.

\footnotetext{
${ }^{1}$ If $t, s$ are two different vectors in a Hilbert space such that $(s \cdot t)=|t||s|$, then $0<|t-s|^{2}=$ $|t|^{2}+|s|^{2}-2(s \cdot t)=|t|^{2}+|s|^{2}-2|s||t|=(|s|-|t|)^{2}$.
} 
Let $g(t):=f(-t)$. The function $g$ satisfies the assumptions of Theorem 2.17. Let $D=N>1, a>0$ and let $\varphi$ be a solution of (2.25) such that $\varphi(r) \rightarrow+\infty$ as $r \rightarrow R$. We set $v(x):=\varphi(|x|)$.

Therefore, the function $v$ satisfies the differential equation

$$
\operatorname{div}\left(|\nabla v|^{p-2} \nabla v\right)=g(v) \quad \text { on } \quad \Omega_{R},
$$

where $\Omega_{R}:=\{x|| x \mid<R\}$. On the other hand the function $U:=-u$ satisfies the inequality

$$
\operatorname{div}\left(|\nabla U|^{p-2} \nabla U\right) \geq g_{1}(U):=g(U) \quad \text { on } \quad \mathbb{R}^{N} .
$$

Since $U(x) \leq v(x)$ for $|x|$ close to $R$ we are in the position to apply the comparison Lemma 2.15. As a consequence, $U(x) \leq v(x)$ for any $x \in \Omega_{R}$. In particular $U(0) \leq v(0)=$ $a$. Letting $a \rightarrow 0$ it follows that $U(0) \leq 0$. Hence $u(0) \geq 0$.

Next, if $f \geq 0$, then $u$ is a nonnegative solution of the inequality, $-\operatorname{div}\left(|\nabla u|^{p-2} \nabla u\right) \geq 0$ on $\mathbb{R}^{N}$. Hence, by the weak Harnack inequality (see [22]) it follows that, either $u \equiv 0$ or $u>0$ on $\mathbb{R}^{N}$.

The argument for proving Theorem 2.3 is the same of the one used in the proof of Theorem 2.1, so we shall be brief.

Proof Theorem 2.3. Let $g(t):=f(-t)$. Under the assumptions of Theorem 2.3, there exists a radial solution $v$ of $\operatorname{div}\left(\frac{\nabla v}{\sqrt{1+|\nabla v|^{2}}}\right) \geq g(v)$ such that $v(0)=a>0$ and $v(r) \rightarrow+\infty$ as $r \rightarrow R$, see [17]. By Lemma 2.15 we get $u(0) \geq-a$. Letting $a \rightarrow 0$, the claim follows.

Proof Theorem 2.4. Let $u$ be a solution of (2.10). The function $v:=u-\alpha$ solves the equation

$$
-\Delta_{p}(v)=f(u-\alpha+\alpha)=f(v+\alpha)=: g(v) .
$$

An application of Theorem 2.1 to the last equation implies $v \geq 0$ i.e. $u \geq \alpha$.

Next, the function $v:=\beta-u$ solves the equation

$$
-\Delta_{p}(v)=-f(u)=-f(-v+\beta)=: g(v) .
$$

Again, we are in the position to apply Theorem 2.1 which yields $v \geq 0$, that is $u \leq \beta$. This concludes the proof.

Proof Theorem 2.5. Step 1. We treat first the case when $f$ has at least a zero. In this case let $\alpha$ be the first zero of $f$. Let $u$ be a solution of (2.7). We set

$$
g(t):=\inf _{s \leq t} f(s), \quad \text { for } t \in \mathbb{R} .
$$


The function $g$ is continuous, non increasing, $g(t)>0$ for $t<\alpha$ and $g(\alpha)=0$.

The function $u$ satisfies the inequality

$$
-\operatorname{div}\left(\frac{\nabla v}{\sqrt{1+|\nabla v|^{2}}}\right) \geq f(u) \geq g(u), \quad \text { on } \mathbb{R}^{N} .
$$

By the change of variable $v:=u-\alpha$ and arguing as in the proof of Theorem 2.4, we get that $u \geq \alpha$. The claim is proved.

Step 2. Next, we assume that $f$ has no zeros. Let $u$ be a solution of (2.7). Let $g$ be the function defined in (2.26). Fix a number $c>0$ and define

$$
g_{c}(t):=\min \{g(t), c-t\}, \quad \text { for } t \in \mathbb{R} .
$$

The function $g_{c}$ is continuous, non increasing, $g_{c}(t)>0$ for $t<c$ and $g_{c}(c)=0$. Since $u$ satisfies

$$
-\operatorname{div}\left(\frac{\nabla v}{\sqrt{1+|\nabla v|^{2}}}\right) \geq f(u) \geq g_{c}(u), \quad \text { on } \mathbb{R}^{N},
$$

by step 1 it follows that $u \geq c$. Since the inequality $u \geq c$ holds for any $c$ we get a contradiction.

Step 3. Let $u$ be a solution of (2.11). By step 1 . we have $u \geq \alpha$. In order to prove the estimate $u \leq \beta$ we consider the function $v:=-u$ and argue as in step 1 .

Proof Theorem 2.8. Arguing by contradiction, let $u$ be a solution of (2.5)). Fix $\alpha \in \mathbb{R}$ and set $v:=u-\alpha$. The function $v$ solves the inequality $-\Delta_{p} v \geq f(u)=f(v+\alpha)$. Since the function $f(\cdot+\alpha)$ satisfies the hypothesis of theorem 2.1 we have $v \geq 0$, that is $u \geq \alpha$. Since the inequality $u \geq \alpha$ holds for any $\alpha$, we obtain $u=+\infty$. This contradiction concludes the proof.

Proof Theorem 2.9. Let $l_{1}:=\lim _{t \rightarrow-\infty} f(t)$ and $l_{2}:=\lim _{t \rightarrow+\infty} f(t)$.

We divide the proof into five steps.

1. We consider first the case $f>0$ and the inequality (2.17). In this case $l_{1}>0$ and Theorem 2.5 yields the thesis.

2. Next assume $f<0$ and let $u$ be a solution of the equation (2.16). Then $v=-u$ satisfies the equation $-\operatorname{div}\left(\frac{\nabla v}{\sqrt{1+|\nabla v|^{2}}}\right)=g(v)$ where $g(t):=-f(-t)$ is a non increasing positive function. This contradicts the step 1 .

Therefore, assume that the function $f$ has at least a zero. Let $u$ be a solution of the equation (2.16). From hypotheses on $f$, the set of its zeros, $S$, is an interval bounded from one side. 
3. Assume, first that $S=\left[\alpha_{1},+\infty\left[\right.\right.$. In this case $l_{1}>0$ and from Theorem 2.5$]$ we have that $u \geq \alpha_{1}$. Hence $f(u(x))=0$ for all $x \in \mathbb{R}^{N}$ and so $u$ is a function bounded from one side which solves the equation

$$
-\operatorname{div}\left(\frac{\nabla u}{\sqrt{1+|\nabla u|^{2}}}\right)=0, \quad \text { on } \mathbb{R}^{N} .
$$

This, by Bombieri-De Giorgi-Miranda Theorem ([2, 12]) implies that $u \equiv \alpha \geq 0$.

4. Next, assume that $\left.S=]-\infty, \alpha_{2}\right]$. By the change of variable $v=-u$ and arguing as in the step 3. we obtain that $u \leq \alpha_{2}$. Hence $u$ is a function bounded from one side which solves (2.27). Therefore $u$ is constant.

5. We analyze the case $S=\left[\alpha_{1}, \alpha_{2}\right]$. In this case $l_{1}>0$ and $l_{2}<0$. By Theorem 2.5 we have that $\alpha_{1} \leq u \leq \alpha_{2}$ that is $u$ takes its value in $S$. Therefore $u$ is a bounded function which solves (2.27). Hence $u$ is constant.

\section{Some extensions of the main results}

We extend our main results to more general quasilinear operator in Section 3.1. In Section 3.2 we extend Theorem 2.1 in Carnot group setting. The final Section 3.3 deals with a quasilinear inequality related to the porous medium equation.

\subsection{A class of differential inequalities}

In this section we shall consider inequalities of the type

$$
-\operatorname{div}(A(|\nabla u|) \nabla u) \geq f(u) \quad \text { on } \quad \mathbb{R}^{N}
$$

where we shall assume that

$$
\left\{\begin{array}{l}
A \in \mathscr{C}(] 0,+\infty[), \quad A(t)>0 \quad \text { for } t>0, \\
t A(|t|) \in \mathscr{C}(\mathbb{R}) \cap \mathscr{C}^{1}(] 0, \infty[) \text { and }(t A(t))^{\prime}>0 \text { for } t>0 .
\end{array}\right.
$$

We shall distinguish two cases accordingly to the asymptotic behavior of the function $t A(t)$. Namely, $\lim _{t \rightarrow+\infty} t A(t)<+\infty$ or $\lim _{t \rightarrow+\infty} t A(t)=+\infty$.

Theorem 3.1 Let $A$ be as in (3.29) and such that $\lim _{t \rightarrow+\infty} t A(t)<+\infty$. Let $f: \mathbb{R} \rightarrow \mathbb{R}$ be a continuous function satisfying (2.3). Let $u$ be a solution of (3.28), then $u \geq 0$ on $\mathbb{R}^{N}$.

We observe that Theorem 2.3 is a particular case of the above theorem. 
In the case $\lim _{t \rightarrow+\infty} t A(t)=+\infty$, then accordingly with [18, 19], we can construct the following function $G$ and $H$. Let $G$ be defined as

$$
G(t):=t^{2} A(t)-\int_{0}^{t} s A(s) d s, \quad t \geq 0 .
$$

The function $G$ is continuous, strictly increasing, $G(0)=0$ and $G(+\infty)=+\infty$, see [18, 19, 17]. Let $H$ be its inverse: the function $H$ is increasing and $H(+\infty)=+\infty$.

Theorem 3.2 Let $A$ be as in (3.29) and such that $\lim _{t \rightarrow+\infty} t A(t)=+\infty$. Let $f: \mathbb{R} \rightarrow \mathbb{R}$ be a continuous function satisfying (2.3) and

$$
\int_{-\infty}^{-1} \frac{1}{H\left(\int_{t}^{-1} f(s) d s\right)} d t<+\infty
$$

Let $u$ be a solution of (3.28), then $u \geq 0$ on $\mathbb{R}^{N}$.

If $A(t)=t^{p-2}$, then $H(t)=\left(\frac{p}{P-1}\right)^{1 / p} t^{1 / p}$ and the above theorem is indeed Theorem 2.1.

We leave the proof of the above results to the interested reader since it is based on the same idea already discussed above by taking into account the nonexistence Theorem 1 and Theorem 2 in [17].

Condition (3.30) is sharp in the following sense. If the integral in (3.30) diverges, then (3.28) admits a negative solution. Indeed from Theorem 3 in [17] it follows that equation (2.25) with $g(t)=f(-t)$ has a positive solution. This implies the claim.

Example 3.3 Let $A(t):=\frac{\ln (1+t)}{t}$ and $f(s) \geq c|s|^{q}$ for $s<0$. We claim that if $q>0$ the solutions of

$$
-\operatorname{div}\left(\ln (1+|\nabla u|) \frac{\nabla u}{|\nabla u|}\right) \geq f(u) \quad \text { on } \quad \mathbb{R}^{N}
$$

are nonnegative. We are in the position to apply Theorem 3.2. In this case $G(t)=t-$ $\ln (1+t)$. In order to prove the claim it is enough to show that the function $H(T)$ behaves at infinity as $T$. Since $H(T)$ is the solution of $t-\ln (1+t)=T$ by the change of variable $z=(1+T)^{-1}$ and $x=(1+t)^{-1}$ the equation becomes $z=\frac{x}{1+x \ln x}$ and we have to study the zero of the function $x(z)$ defined implicitly. It is easy to recognize that $x(z)=z+o(z)$, and this implies the claim.

Using the same argument as above one can prove the following. We omit the details.

Theorem 3.4 Let $f: \mathbb{R} \rightarrow \mathbb{R}$ be a continuous function such that : there exists $c>0$ and $q>1$ such that $f(s) \geq c(\ln (1+|s|))^{q}$ for $s<0$. Then the solutions of (3.31) are nonnegative. 
The analogous results of Theorem 2.4, 2.5, 2.6, 2.8, 2.9 are the following.

Theorem 3.5 Let $A$ be as in (3.29) and such that $\lim _{t \rightarrow+\infty} t A(t)=+\infty$. Let $f: \mathbb{R} \rightarrow \mathbb{R}$ be a continuous function such that there exists $\alpha, \beta \in \mathbb{R}, \alpha \leq \beta$ such that

$f_{]-\infty, \alpha[}$ is positive and non increasing, $f_{\beta,+\infty[}$ is negative and non increasing,

and

$$
\int_{-\infty}^{\alpha} \frac{1}{H\left(\int_{t}^{\alpha} f(s) d s\right)} d t<+\infty, \quad \int_{\beta}^{\infty} \frac{1}{H\left(\int_{\beta}^{t}-f(s) d s\right)} d t<+\infty .
$$

Let $u$ be a solution of

$$
-\operatorname{div}(A(|\nabla u|) \nabla u)=f(u) \quad \text { on } \quad \mathbb{R}^{N},
$$

then $u$ is bounded and $\alpha \leq u(x) \leq \beta$ for any $x \in \mathbb{R}^{N}$.

Theorem 3.6 Let $A$ be as in (3.29) and such that $\lim _{t \rightarrow+\infty} t A(t)<+\infty$. Let $f: \mathbb{R} \rightarrow \mathbb{R}$ be a continuous function such that

$$
\liminf _{t \rightarrow-\infty} f(t)>0
$$

If $u$ is a solution of (3.28), then $f$ has at least a zero, and set $\alpha:=$ the first zero of $f$ (that is $\alpha:=\min S$ where $\left.S:=f^{-1}(0)\right)$ we have $u \geq \alpha$. In particular if $f>0$ the (3.28) has no solution.

Moreover if

$$
\limsup _{t \rightarrow+\infty} f(t)<0
$$

and $u$ solves 3.34 then $u$ is bounded and $\alpha \leq u(x) \leq \beta$ for any $x \in \mathbb{R}^{N}$, where $\beta:=$ last zero of $f$ (that is $\beta:=\max S$ ).

Theorem 3.7 Let $A$ be as in (3.29) and such that $\lim _{t \rightarrow+\infty} t A(t)=+\infty$. Let $f: \mathbb{R} \rightarrow \mathbb{R}$ be a non increasing continuous function such that

$$
f(t)>0 \text { if } t<0 \text {, and } f(t)<0 \text { if } t>0
$$

and (3.33) holds with $\alpha=-1$ and $\beta=1$.

If $u$ be a solution of (3.34), then $u \equiv 0$ on $\mathbb{R}^{N}$.

Theorem 3.8 Let $A$ be as in (3.29) and such that $\lim _{t \rightarrow+\infty} t A(t)=+\infty$. Let $f: \mathbb{R} \rightarrow \mathbb{R}$ be a positive, non increasing, continuous function satisfying (3.30). Then the inequality (3.28) has no solutions. 
Theorem 3.9 Let $A$ be as in (3.29) and such that $\lim _{t \rightarrow+\infty} t A(t)<+\infty$. Let $f: \mathbb{R} \rightarrow \mathbb{R}$ be a non increasing, continuous function. If $u$ is a solution of (3.34) then $u$ solves the homogeneous problem

$$
-\operatorname{div}(A(|\nabla u|) \nabla u)=0 \quad \text { on } \quad \mathbb{R}^{N},
$$

and for all $x \in \mathbb{R}^{N}, u(x) \in S$ where $S:=f^{-1}(0)$. In particular if $f(t) \neq 0$ for any $t$, then (3.34) has no solutions.

In addition, if $f$ is supposed to be positive then the inequality (3.28) has no solutions.

Remark 3.10 Very recently, by using a completely different technique, a strong generalization of Theorem 3.9 has been obtained by James Serrin [23, Theorem 3.23]. We wish to thank Alberto Farina for pointing out this information.

For recent nonexistence results related to anticoercive problems, we refer the interested reader to the forthcoming paper [8].

\subsection{Inequalities on Carnot Groups}

Let $\mathbb{R}^{N} \equiv \mathbb{G}$ be a Carnot group and let $\nabla_{L}$ be the horizontal gradient on $G$ and $Q>1$ the homogeneous dimension (see Appendix and [1] for details on these structures). Let $\Gamma_{p}$ be the fundamental solution of the quasilinear operator $\Delta_{L, p} u=\operatorname{div}_{L}\left(\left|\nabla_{L} u\right|^{p-2} \nabla_{L} u\right)$ at the origin. Set

$$
N_{p}:= \begin{cases}\Gamma_{p}^{\frac{p-1}{p-Q}} & p>1, p \neq Q \\ \exp \left(-\Gamma_{p}\right) & p=Q\end{cases}
$$

It is known that $N_{p}$ is a homogeneous norm on $\mathbb{G}$. From [4, 5], it is known that $N_{p}$ is Hölder continuous. In what follows we shall assume that $N_{p}$ is smooth. This assumption is satisfied for example for "Heisenberg type" groups. See [5].

With the above notation, we have that if $\zeta: \mathbb{R} \rightarrow \mathbb{R}$ is a smooth function, then the radial function $v:=\zeta \circ N_{p}: \mathbb{G} \rightarrow \mathbb{R}$ satisfies

$$
\Delta_{G, p} v:=\operatorname{div}_{L}\left(\left|\nabla_{L} v\right|^{p-2} \nabla_{L} v\right)=(p-1) \psi^{p}\left|\zeta^{\prime}\right|^{p-2}\left(\zeta^{\prime \prime}(r)+\frac{Q-1}{p-1} \frac{\zeta^{\prime}(r)}{r}\right)_{r=N_{P}},
$$

where $\psi:=\left|\nabla_{L} N_{p}\right|$ is a bounded function, see [6]. Hence we can apply the same arguments used in the preceding section obtaining an analog of Theorem 2.1 in this more general setting.

Theorem 3.11 Let $p>1$. Let $f: \mathbb{R} \rightarrow \mathbb{R}$ be a continuous function satisfying (2.3) and (2.4). Let $u$ be a solution of

$$
-\operatorname{div}_{L}\left(\left|\nabla_{L} u\right|^{p-2} \nabla_{L} u\right) \geq f(u) \text { on } \quad \mathbb{R}^{N},
$$

then $u \geq 0$ on $\mathbb{R}^{N}$. Moreover if $f(t) \geq 0$ for $t \geq 0$ then, either $u \equiv 0$ or $u>0$ on $\mathbb{R}^{N}$. 
Immediate consequences of Theorem 3.11 are the following Liouville type theorems.

Theorem 3.12 Let $p>1$. Let $f: \mathbb{R} \rightarrow \mathbb{R}$ be a continuous function such that there exists $\alpha, \beta \in \mathbb{R}, \alpha \leq \beta$ such that (2.8) and (2.9) hold. If $u$ is a solution of

$$
-\operatorname{div}_{L}\left(\left|\nabla_{L} u\right|^{p-2} \nabla_{L} u\right)=f(u) \quad \text { on } \quad \mathbb{R}^{N},
$$

then $u$ is bounded and $\alpha \leq u(x) \leq \beta$ for any $x \in \mathbb{R}^{N}$. In particular, if $q>p-1$ and $u$ is a solution of

then $u \equiv 0$ on $\mathbb{R}^{N}$.

$$
\Delta_{G, p} u=|u|^{q-1} u \quad \text { on } \quad \mathbb{R}^{N},
$$

Remark 3.13 The conclusion related to inequality (3.37) in the Euclidean setting, $p=2$, and $f(u)=|u|^{q-1} u$ has been obtained by Brezis [3] by using a variant of Kato's inequality and .

Theorem 3.14 Let $p>1$. Let $f: \mathbb{R} \rightarrow \mathbb{R}$ be a positive, non increasing, continuous function satisfying (2.4). Then the inequality (3.37) has no solutions.

Corollary 3.15 Let $p \geq Q>1$ and $f: \mathbb{R} \rightarrow[0,+\infty[$ be a continuous function satisfying (2.3) and (2.4). If $u$ is a solution of (2.5) then $u$ is constant on $\mathbb{R}^{N}$. More precisely $u \equiv \alpha \geq 0$ and $f(\alpha)=0$.

Moreover if $f(t)>0$ for $t \geq 0$, then (2.5) has no solutions.

Proof Theorem 3.11. Let $u$ be a solution of (2.5). Since the inequality is invariant under translations, it is sufficient to prove that $u(0) \geq 0$.

Let $C>0$ be a constant such that $\psi^{p} \leq C$. Set $g(t):=f(-t) / C$. The function $g$ satisfies the assumptions of Theorem 2.17. Let $D=Q>1$ be the homogeneous dimension. Let $a>0$ and let $\varphi$ be a solution of (2.25) such that $\varphi(r) \rightarrow+\infty$ as $r \rightarrow R$. We set $v(x):=\varphi\left(N_{p}(x)\right)$. By computation we have,

$$
\begin{array}{r}
\operatorname{div}_{L}\left(\left|\nabla_{L} v\right|^{p-2} \nabla_{L} v\right)=(p-1) \psi^{p}\left|v^{\prime}\right|^{p-2}\left(v^{\prime \prime}(r)+\frac{Q-1}{p-1} \frac{v^{\prime}(r)}{r}\right)_{r=N_{p}} \\
=\psi^{p} N_{p}^{1-Q}\left(r^{Q-1}\left|v^{\prime}(r)\right|^{p-2} v^{\prime}(r)\right)_{r=N_{p}}^{\prime} .
\end{array}
$$

Therefore, the function $v$ satisfies the differential equation

$$
\operatorname{div}_{L}\left(\left|\nabla_{L} v\right|^{p-2} \nabla_{L} v\right)=g_{2}(x, v):=\psi^{p} g(v) \leq C g(v)
$$

on $\Omega_{R}:=\left\{x \mid N_{p}(x)<R\right\}$. On the other hand the function $U:=-u$ satisfies the inequality

$$
\operatorname{div}_{L}\left(\left|\nabla_{L} U\right|^{p-2} \nabla_{L} U\right) \geq f(-U)=C g(U)=: g_{1}(U) \quad \text { on } \quad \mathbb{R}^{N}
$$


Since $g_{1} \geq g_{2}$ and $U(x) \leq v(x)$ for $N_{p}(x)$ close to $R$ we are in the position to apply the comparison Lemma 2.15. As a consequence, $U(x) \leq v(x)$ for any $x \in \Omega_{R}$. In particular $U(0) \leq v(0)=a$. Letting $a \rightarrow 0$ it follows that $U(0) \leq 0$. Hence $u(0) \geq 0$.

Next, if $f \geq 0$, then $u$ is nonnegative and solves the inequality $-\operatorname{div}_{L}\left(\left|\nabla_{L} u\right|^{p-2} \nabla_{L} u\right) \geq 0$ on $\mathbb{R}^{N}$. Hence, by the weak Harnack inequality (see [4]) it follows that, either $u \equiv 0$ or $u>0$ on $\mathbb{R}^{N}$.

The proofs of the above Liouville theorems are very similar to those given in Section 2. so we omit them. The proof of Corollary 3.15 relies the fact that the only nonnegative functions $u$ such that $-\Delta_{G, p} u \geq 0$ on $\mathbb{R}^{N}$ with $Q \leq p$ are the constants, see [7].

\subsection{A porous medium type inequality}

We end this paper by pointing out the following slight modification of Theorem 2.1.

Theorem 3.16 Let $\gamma \geq 1$. Let $f: \mathbb{R} \rightarrow \mathbb{R}$ be a continuous function satisfying (2.3) and

$$
\int_{-\infty}^{-1}|t|^{\gamma-1}\left(\int_{t}^{-1} f(s)|s|^{\gamma-1} d s\right)^{-\frac{1}{2}} d t<+\infty .
$$

Let $u$ be a solution of

$$
-\Delta\left(|u|^{\gamma-1} u\right) \geq f(u) \quad \text { on } \mathbb{R}^{N}
$$

then $u \geq 0$ on $\mathbb{R}^{N}$.

In particular if $u$ is a solution and $f(t) \geq C|t|^{q}$ for $t<0$ with $q>\gamma$, then either $u \equiv 0$ or $u>0$ on $\mathbb{R}^{N}$.

\section{Appendix}

We quote some facts on Carnot groups and refer the interested reader to [1, 15] for more detailed information on this subject.

A Carnot group is a connected, simply connected, nilpotent Lie group $\mathbb{G}$ of dimension $N$ with graded Lie algebra $\mathcal{G}=V_{1} \oplus \cdots \oplus V_{r}$ such that $\left[V_{1}, V_{i}\right]=V_{i+1}$ for $i=1 \ldots r-1$ and $\left[V_{1}, V_{r}\right]=0$. Such an integer $r$ is called the step of the group. We set $l=n_{1}=\operatorname{dim} V_{1}$, $n_{2}=\operatorname{dim} V_{2}, \ldots, n_{r}=\operatorname{dim} V_{r}$. A Carnot group $\mathbb{G}$ of dimension $N$ can be identified, up to an isomorphism, with the structure of a homogeneous Carnot Group $\left(\mathbb{R}^{N}, \circ, \delta_{R}\right)$ defined as follows; we identify $\mathbb{G}$ with $\mathbb{R}^{N}$ endowed with a Lie group law $\circ$. We consider $\mathbb{R}^{N}$ split in $r$ subspaces $\mathbb{R}^{N}=\mathbb{R}^{n_{1}} \times \mathbb{R}^{n_{2}} \times \cdots \times \mathbb{R}^{n_{r}}$ with $n_{1}+n_{2}+\cdots+n_{r}=N$ and $\xi=\left(\xi^{(1)}, \ldots, \xi^{(r)}\right)$ with $\xi^{(i)} \in \mathbb{R}^{n_{i}}$. We shall assume that for any $R>0$ the dilation $\delta_{R}(\xi)=\left(R \xi^{(1)}, R^{2} \xi^{(2)}, \ldots, R^{r} \xi^{(r)}\right)$ is a Lie group automorphism. The Lie algebra of leftinvariant vector fields on $\left(\mathbb{R}^{N}, \circ\right)$ is $\mathcal{G}$. For $i=1, \ldots, n_{1}=l$ let $X_{i}$ be the unique vector field 
in $\mathcal{G}$ that coincides with $\partial / \partial \xi_{i}^{(1)}$ at the origin. We require that the Lie algebra generated by $X_{1}, \ldots, X_{l}$ is the whole $\mathcal{G}$.

We denote with $\nabla_{L}$ the vector field $\nabla_{L}:=\left(X_{1}, \ldots, X_{l}\right)^{T}$ and we call it horizontal vector field. Moreover, the vector fields $X_{1}, \ldots, X_{l}$ are homogeneous of degree 1 with respect to $\delta_{R}$ and in this case $Q=\sum_{i=1}^{r} i n_{i}=\sum_{i=1}^{r} i \operatorname{dim} V_{i}$ is called the homogeneous dimension of $\mathbb{G}$. The canonical sub-Laplacian on $\mathbb{G}$ is the second order differential operator defined by $\Delta_{G}=\sum_{i=1}^{l} X_{i}^{2}$ and for $p>1$ the $p$-sub-Laplacian operator is $\sum_{i=1}^{l} X_{i}\left(\left|\nabla_{L} u\right|^{p-2} X_{i} u\right)$. Since $X_{1}, \ldots, X_{l}$ generate the whole $\mathcal{G}$, the sub-Laplacian $\Delta_{G}$ satisfies the Hörmander hypoellipticity condition.

A nonnegative continuous function $N: \mathbb{R}^{N} \rightarrow \mathbb{R}_{+}$is called a homogeneous norm on $\mathbb{G}$, if $N\left(\xi^{-1}\right)=N(\xi), N(\xi)=0$ if and only if $\xi=0$, and it is homogeneous of degree 1 with respect to $\delta_{R}$ (i.e. $N\left(\delta_{R}(\xi)\right)=R N(\xi)$ ). A homogeneous norm $N$ defines on $\mathbb{G}$ a pseudo-distance defined as $d(\xi, \eta):=N\left(\xi^{-1} \eta\right)$, which in general is not a distance. If $N$ and $\tilde{N}$ are two homogeneous norms, then they are equivalent, that is, there exists a constant $C>0$ such that $C^{-1} N(\xi) \leq \tilde{N}(\xi) \leq C N(\xi)$. Let $N$ be a homogeneous norm, then there exists a constant $C>0$ such that $C^{-1}|\xi| \leq N(\xi) \leq C|\xi|^{1 / r}$, for $N(\xi) \leq 1$. An example of homogeneous norm is $N(\xi):=\left(\sum_{i=1}^{r}\left|\xi_{i}\right|^{2 r ! / i}\right)^{1 / 2 r !}$.

Notice that if $N$ is a homogeneous norm differentiable a.e., then $\left|\nabla_{L} N\right|$ is homogeneous of degree 0 with respect to $\delta_{R}$; hence $\left|\nabla_{L} N\right|$ is bounded.

Special examples of Carnot groups are the Euclidean spaces $\mathbb{R}^{Q}$. Moreover, if $Q \leq 3$ then any Carnot group is the ordinary Euclidean space $\mathbb{R}^{Q}$.

The most simple nontrivial example of a Carnot group is the Heisenberg group $\mathbb{H}^{1}=\mathbb{R}^{3}$. For an integer $n \geq 1$, the Heisenberg group $\mathbb{H}^{n}$ is defined as follows: let $\xi=\left(\xi^{(1)}, \xi^{(2)}\right)$ with $\xi^{(1)}:=\left(x_{1}, \ldots, x_{n}, y_{1}, \ldots, y_{n}\right)$ and $\xi^{(2)}:=t$. We endow $\mathbb{R}^{2 n+1}$ with the group law $\hat{\xi} \circ \tilde{\xi}:=\left(\hat{x}+\tilde{x}, \hat{y}+\tilde{y}, \hat{t}+\tilde{t}+2 \sum_{i=1}^{n}\left(\tilde{x}_{i} \hat{y}_{i}-\hat{x}_{i} \tilde{y}_{i}\right)\right)$. We consider the vector fields

$$
X_{i}:=\frac{\partial}{\partial x_{i}}+2 y_{i} \frac{\partial}{\partial t}, Y_{i}:=\frac{\partial}{\partial y_{i}}-2 x_{i} \frac{\partial}{\partial t}, \quad \text { for } i=1, \ldots, n,
$$

and the associated Heisenberg gradient $\nabla_{H}:=\left(X_{1}, \ldots, X_{n}, Y_{1}, \ldots, Y_{n}\right)^{T}$. The Kohn Laplacian $\Delta_{H}$ is then the operator defined by $\Delta_{H}:=\sum_{i=1}^{n} X_{i}^{2}+Y_{i}^{2}$. The family of dilations is given by $\delta_{R}(\xi):=\left(R x, R y, R^{2} t\right)$ with homogeneous dimension $Q=2 n+2$. In $\mathbb{H}^{n}$ a canonical homogeneous norm is defined as $|\xi|_{H}:=\left(\left(\sum_{i=1}^{n} x_{i}^{2}+y_{i}^{2}\right)^{2}+t^{2}\right)^{1 / 4}$.

Acknowledgements We wish to thank Alberto Farina and James Serrin for useful discussions and for attracting our attention to references [3], [13], [14], [23]. 


\section{References}

[1] A. Bonfiglioli, E. Lanconelli and F. Uguzzoni. Stratified Lie Groups and Potential Theory for their Sub-Laplacians, Springer Monograph in Mathematics XXVI Springer-Verlag Berlin-Heidelberg, (2007) 800 pages.

[2] E. Bombieri, E. De Giorgi and L. Miranda. Una maggiorazione a priori per le superfici minimali non parametriche, Arch. Rational Mech. 32 (1969), 255-267.

[3] H. Brezis. Semilinear equations in $\mathbb{R}^{N}$ without conditions at infinity, Appl. Math. Optimizations 12 (1984), 271-282.

[4] L. Capogna, D. Danielli and N. Garofalo. An embedding theorem and the Harnack inequality for nonlinear subelliptic equations, Comm. Partial Differential Equations 18 (1993), no. 9-10, 1765-1794.

[5] L. Capogna, D. Danielli and N. Garofalo. Capacitary estimates and the local behavior of solutions of nonlinear subelliptic equations, Amer. J. Math. 118 (1996), no. $6,1153-1196$.

[6] L. D'Ambrosio. Hardy-type inequalities related to degenerate elliptic differential operators, Ann. Scuola Norm. Sup. Pisa Cl. Sci. Ser. 5 IV (2005), 451-486.

[7] L. D'Ambrosio. Liouville theorems for anisotropic quasilinear inequalities, Nonlinear Anal. TMA 70 (2008), 2855-2869.

[8] L. D'Ambrosio and E. Mitidieri. A priori estimates, positivity results, and nonexistence theorems for quasilinear degenerate elliptic inequalities , 1-55 Submitted (2009).

[9] L. D'Ambrosio and E. Mitidieri. Positivity property of solutions of some quasilinear elliptic inequalities, Functional Analysis and Evolution Equations, The Günter Lumer Volume, Birkhäuser, (2008), 147-156.

[10] L. D'Ambrosio, E. Mitidieri and S. I. Pohozaev. Representation Formulae and Inequalities for Solutions of a Class of Second Order Partial Differential Equations, Trans. Amer. Math. Soc. 358 (2006), 893-910.

[11] E. Mitidieri and S. I. Pohozaev. Some Generalizations of Bernstein Theorem, Differential Equations 38 (2002), no. 3, 373-378.

[12] E. De Giongi. Maggiorazioni a priori relative ad superfici minimali , Atti del Convegno di Analisi Funzionale Roma (1968) - Symposia Mathematica II Academic Press (1969), 283-285. 
[13] A. Farina . Liouville-type Theorems for elliptic problem, Handbook of Differential Equations : Stationary Partial Differential Equations Equations. Vol. 4, Edited by M.Chipot, Elsevier B.V, 61-116 (2007).

[14] A. Farina and J. Serrin. Entire solutions of completely coercive quasilinear elliptic equations, preprint 1-46 (2009).

[15] G. B. Folland and E. M. Stein. Hardy spaces on homogeneous groups, volume 28 of Mathematical Notes. Princeton University Press, Princeton, N.J. (1982).

[16] E. Mitidieri and S. I. Pohozaev. Positivity property of solutions of some elliptic inequalities on $\mathbf{R}^{n}$, Doklady Mathematics 68 (2003), no. 3, 159-164.

[17] Y. Naito and H. Usami. Entire solutions of the inequality $\operatorname{div}(A(|D u|) D u) \geq f(u)$, Math. Z. 225 (1997), no. 1, 167-175.

[18] W.-M. Ni and J. SerRin. Non-existence theorems for quasilinear partial differential equations, Supplemento di Rendiconti Circolo Matematico di Palermo 8 (1985), 171185 .

[19] W.-M. Ni and J. SerRin. Existence and non-existence theorems for ground states of quasilinear partial differential equations. The anomalous case, Acc. Naz. dei Lincei, Atti dei Convegni 77 (1986), 231-257.

[20] R. Osserman. On the inequality $\Delta u \geq f(u)$, Pacific J. Math. 7 (1957), 1641-1647.

[21] P. Pucci and J. Serrin. The Maximum Principle, Progress in Nonlinear Differential Equations and Their Applications 73, Birkhäuser Basel-Boston-Berlin (2007) X-234 pages.

[22] J. Serrin. Local behavior of solutions of quasilinear elliptic equations, Acta Math. 111 (1964), 247-302.

[23] J. Serrin. Entire solutions of quasilinear elliptic equations, J. Math. Anal. Appl., 352 (2009), 3-14. 